\title{
A CENSUS OF AM CVn STARS: THREE NEW CANDIDATES AND ONE CONFIRMED 48.3-MINUTE BINARY
}

\author{
A. RaU ${ }^{1,2}$, G. H. A. Roelofs ${ }^{3}$, P. J. Groot ${ }^{4}$, T. R. Marsh ${ }^{5}$, G. Nelemans ${ }^{4}$, D. Steeghis ${ }^{3,5}$, M. Salvato 2,6 , \\ AND M. M. KASLIWAL ${ }^{2}$ \\ ${ }^{1}$ Max-Planck Institute for Extraterrestrial Physics, Giessenbachstr. 1, Garching 85748, Germany; arau@mpe.mpg.de \\ ${ }^{2}$ Caltech Optical Observatories, MS 105-24, California Institute of Technology, Pasadena, CA 91125, USA \\ ${ }^{3}$ Harvard-Smithsonian Center for Astrophysics, 60 Garden Street, Cambridge, MA 02138, USA \\ ${ }^{4}$ Department of Astrophysics, IMAPP, Radboud University Nijmegen, P.O. Box 9010, 6500 GL Nijmegen, The Netherlands \\ ${ }^{5}$ Department of Physics, University of Warwick, Coventry CV4 7Al, UK \\ ${ }^{6}$ Max-Planck-Institute for Plasmaphysics, Boltzmannstr. 2, Garching 85748, Germany \\ Received 2009 September 23; accepted 2009 November 11; published 2009 December 11
}

\begin{abstract}
We present three new candidate AM CVn binaries, and one confirmed new system, from a spectroscopic survey of color-selected objects from the Sloan Digital Sky Survey (SDSS). All four systems were found from their helium emission lines in low-resolution spectra taken on the Hale telescope at Palomar, the Nordic Optical Telescope, and the William Herschel Telescope on La Palma. The ultra-compact binary nature of SDSS J090221.35+381941.9 was confirmed using phase-resolved spectroscopy at the Keck-I telescope. From the characteristic radial velocity "S-wave" observed in the helium emission lines, we measure an orbital period of $48.31 \pm 0.08$ minutes. The continuum emission can be described with a blackbody or a helium white dwarf atmosphere of $T_{\text {eff }} \sim 15,000 \mathrm{~K}$, in agreement with theoretical cooling models for relatively massive accretors and/or donors. The absence in the spectrum of broad helium absorption lines from the accreting white dwarf suggests that the accreting white dwarf cannot be much hotter than $15,000 \mathrm{~K}$, or that an additional component such as the accretion disk contributes substantially to the optical flux. Two of the candidate systems, SDSS J152509.57+360054.5 and SDSS J172102.48+273301.2, do show helium absorption in the blue part of their spectra in addition to the characteristic helium emission lines. This in combination with the high effective temperatures of $\sim 18,000 \mathrm{~K}$ and $\sim 16,000 \mathrm{~K}$ suggests both to be at orbital periods below $\approx 40$ minutes. The third candidate, SDSS J164228.06+193410.0, exhibits remarkably strong helium emission on top of a relatively cool $\left(T_{\text {eff }} \sim 12,000 \mathrm{~K}\right)$ continuum, indicating an orbital period above $\sim 50$ minutes.
\end{abstract}

Key words: accretion, accretion disks - binaries: close - novae, cataclysmic variables - stars: individual (SDSS J090221.34+381941.9, SDSS J152509.57+360054.5, SDSS J164228.06+193410.0, SDSS J172102.48+273301.2) - white dwarfs

\section{INTRODUCTION}

The AM CVn stars form a small class of interacting binaries, consisting of white dwarf accretors with degenerate or semidegenerate helium-transferring companion stars. AM CVn stars exist in ultra-compact configurations, with observed orbital periods, $P_{\text {orb }}$, ranging from 65 minutes down to 10 minutes (below the orbital period minimum for hydrogen-rich donors, see Nelemans 2005, for a recent review). Two ultrashort-period candidates, V407 Vul (Cropper et al. 1998) and HM Cnc (Israel et al. 2002), possibly extend the $P_{\mathrm{orb}}$-distribution further down to 5.3 minutes.

AM CVn stars are of interest for binary stellar evolution theory as they form the end product of several evolutionary scenarios (e.g., Nelemans et al. 2001; Podsiadlowski et al. 2003) and can potentially produce rare, sub-luminous, SN Ia-like explosions (SN.Ia; Bildsten et al. 2007). They are also the strongest known sources that can be detected as gravitational-wave emitters with the future Laser Interferometer Space Antenna (LISA; see, e.g., Nelemans et al. 2004; Stroeer et al. 2005; Roelofs et al. 2007d).

Our understanding of the physics of these systems has increased significantly over the last few years (e.g., Deloye et al. 2005; Bildsten et al. 2006; Deloye et al. 2007), while observationally, the Sloan Digital Sky Survey (SDSS; York et al. 2000) has been instrumental. Pre-SDSS, only $\sim 10$ AM CVn binaries were known (Roelofs et al. 2005), and the extreme heterogeneity of this already small sample made a census of the Galactic population of AM CVn stars impossible. The SDSS-I
(Data Release 5) provided a substantially more homogeneous sample of six new AM CVn stars, allowing for a local space density estimate, limited by the still small size of the sample (Roelofs et al. 2007d).

Noting that the AM CVn stars occupy a relatively sparsely populated region in color space where the spectroscopic completeness down to $g=20.5$ in the SDSS database is only $\sim 20 \%$, we started an extensive spectroscopic survey program at a number of observatories worldwide to resolve the "hidden" population of AM CVn stars in the SDSS photometry. The aim of this project is to significantly increase the total known population of AM CVn stars and, more importantly, enlarge the homogeneous sample of these sources. Ultimately, the space density of AM $\mathrm{CVn}$ stars, and further population characteristics such as their orbital period distribution, will allow comparisons with predictions from theoretical models, and will allow better modeling of the Galactic gravitational-wave foreground signal seen by LISA.

Details of our spectroscopic follow-up program, in particular the sample selection criteria, and the first (peculiar) new AM CVn star (SDSS J080449.49+161624.8) were described in Roelofs et al. (2009). A complete catalog of sources, and a detailed AM CVn population study, will be presented when the spectroscopic survey program is complete. Here, we give a status update after the first year and present four new discoveries, including a detailed spectroscopic study of one confirmed new AM CVn star.

The outline of the paper is as follows. In Section 2, we summarize the observations and data reduction. We present the new AM CVn candidates as well as the spectral and timing 

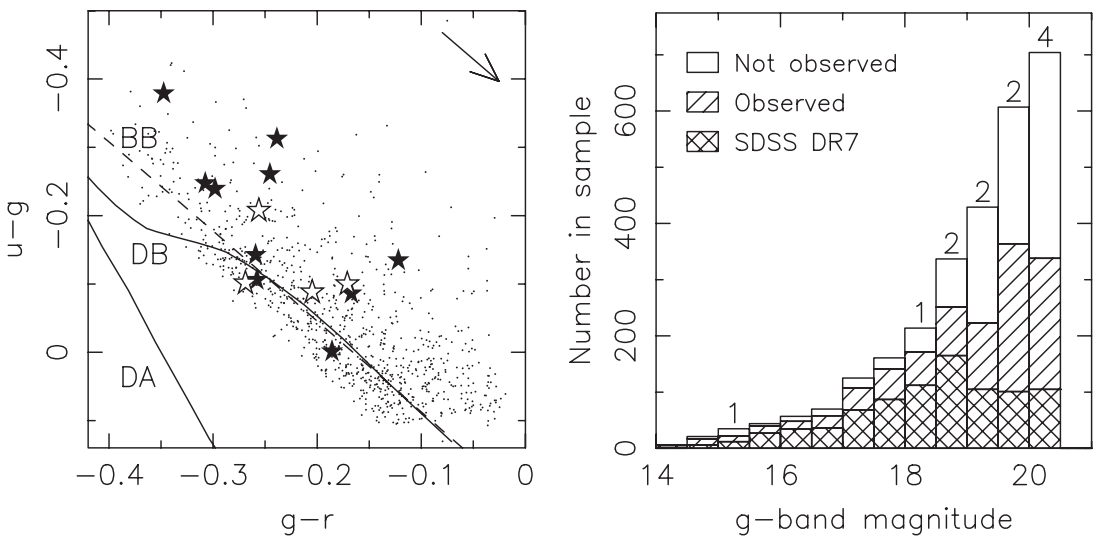

Figure 1. Photometric colors (left, dereddened) and spectroscopic completeness (right, not dereddened) of our sample. Left: stars indicate known AM CVn stars with SDSS photometry; open stars are the four new systems presented here (SDSS J0902, J1525, J1721, J1642 from left to right). The dots denote our candidate population up to DR7. Black-body (BB), helium-atmosphere (DB) and hydrogen-atmosphere (DA) white dwarf cooling curves are indicated. The arrow shows the reddening vector for a $g$-band extinction of 0.2 mag. Right: numbers above bins give the number of AM CVns in our spectroscopy or the DR7 spectroscopic database. Two systems with DR7 photometry have not been observed spectroscopically by us or the SDSS. The brightest system is the well-studied source GP Com.

analysis of the confirmed system in Section 3. Our conclusions are given in Section 4.

\section{OBSERVATIONS AND DATA REDUCTION}

Low-resolution, low-signal-to-noise ratio spectra of objects in our color-selected sample have been obtained on a multitude of telescopes. To date, almost half of our sample of $\approx 1500$ candidates have been completed (see Figure 1 for a concise summary of the current statistics). Two of the systems presented here (SDSS J172102.48+273301.2, hereafter SDSS J1721, and SDSS J152509.57+360054.5, hereafter SDSS J1525) were identified as helium-emission-line objects from spectra taken at the $2.6 \mathrm{~m}$ Nordic Optical Telescope (NOT) on La Palma, Spain, with the ALFOSC spectrograph fitted with the grism 11 (see Table 1). Slits with $0{ }^{\prime} 9$ and 1".3 width were used for SDSS J1721 and SDSS J1525, respectively, offering resolutions of $R \sim 210$ and $R \sim 145$ at $5200 \AA$. The third object, SDSS J164228.06+193410.0 (hereafter SDSS J1642), was identified in a spectrum taken at the $4.2 \mathrm{~m}$ William Herschel Telescope (WHT), also on La Palma, equipped with the Auxiliary-port Camera (ACAM) and the $400 \mathrm{~V}$ holographic grism, providing an effective resolution $R \sim 450$ at $6000 \AA$. This setup was also used to obtain confirmation spectra of the two objects discovered at the NOT. The NOT and WHT spectra were corrected for Galactic foreground extinction assuming that the AM CVn stars are on average located behind the Galactic plane dust screen (Roelofs et al. 2009).

The identifying spectrum of SDSS J090221.35+381941.9 (hereafter SDSS J0902) was taken at the Hale $5 \mathrm{~m}$ telescope at Palomar Observatory equipped with the Double-Beam Spectrograph (DBSP; Oke \& Gunn 1982). A 1".5 slit and the 300/ 3990 and $316 / 7500$ gratings were used, achieving a spectral resolution on the blue and red sides of $R \sim 540(4000 \AA)$ and $R \sim 700$ (6000 ̊), respectively.

Follow-up phase-resolved spectroscopy of SDSS J0902 was obtained on 2009 January 26 and 27 using the Low-Resolution Imager and Spectrograph (LRIS; Oke et al. 1995) at the 10- $\mathrm{m}$ Keck-I telescope (see Table 1). Similar to DBSP, LRIS is a dualbeam instrument. We used the 600/4000 grism and 600/7500 grating for the blue and red arms, respectively, with the light split by a dichroic at $\approx 5600 \AA$. The blue arm data were binned on the chip by factors of 4 each in spatial and dispersion direction. This allowed a reduction of the overhead due to $\mathrm{CCD}$ readout from
Table 1

Log of Spectroscopic Observations

\begin{tabular}{clcc}
\hline \hline SDSS . . & \multicolumn{1}{c}{ UT Date } & Setup & Exposures \\
\hline J090221.35+381941.9 & 2009 Jan 1 & P200/DBSP & $1 \times 910 \mathrm{~s}$ \\
& 2009 Jan 26 & Keck-I/LRIS & $20 \times 240 \mathrm{~s}$ \\
& 2009 Jan 27 & Keck-I/LRIS & $45 \times 180 \mathrm{~s}$ a \\
$\mathrm{J} 152509.57+360054.5$ & 2009 May 26 & NOT/ALFOSC & $1 \times 600 \mathrm{~s}$ \\
& 2009 Jun 25 & WHT/ACAM & $1 \times 900 \mathrm{~s}$ \\
$\mathrm{~J} 164228.06+193410.0$ & 2009 Jun 23 & WHT/ACAM & $2 \times 400 \mathrm{~s}$ \\
$\mathrm{~J} 172102.48+273301.2$ & 2009 May 25 & NOT/ALFOSC & $1 \times 600 \mathrm{~s}$ \\
& 2009 Jun 19 & WHT/ACAM & $1 \times 600 \mathrm{~s}$ \\
\hline
\end{tabular}

Note. ${ }^{\text {a }} 43$ exposures on the red side.

$42 \mathrm{~s}$ (unbinned) to $27 \mathrm{~s}$. All LRIS observations were performed with a long slit of 1..5 width and the atmospheric dispersion corrector in place. Typical seeing was about 1 1.2 resulting in an effective spectral resolution of about $4.5 \AA$ full-width at half-maximum (FWHM) at $4500 \AA$, or $300 \mathrm{~km} \mathrm{~s}^{-1}$. The red arm data were binned $2 \times 2$ providing readout times of $34 \mathrm{~s}$ (compared to $72 \mathrm{~s}$ unbinned) and a resolution of $4.4 \AA$ at $6000 \AA$ $\left(\approx 220 \mathrm{~km} \mathrm{~s}^{-1}\right)$.

All data were reduced with standard IRAF routines, and spectra were extracted using an optimal (variance-weighted) method. Spectro-photometric flux calibration was carried out with spectra of the standard star Feige 34 (Oke 1990) taken each night. Correction for Galactic foreground extinction of $E(B-V)=0.024 \mathrm{mag}$ (Schlegel et al. 1998) was applied.

\section{RESULTS}

\subsection{Identification Spectra of the New AM CVns}

The extracted spectra of all four objects are shown in Figure 2. The prominent neutral He emission lines are clearly recognized in all of them, most notably He I 5876, 6678, and 7065. This, and the absence of hydrogen in the spectra, suggests that all four objects are likely new members of the class of AM CVn stars, although further observations are needed to confirm their ultra-compact binary nature beyond question.

\subsection{Phase-resolved Spectroscopy of SDSS J0902}

Phase-resolved spectroscopy is the technique of choice for measuring the orbital period of AM CVn binaries, for which 


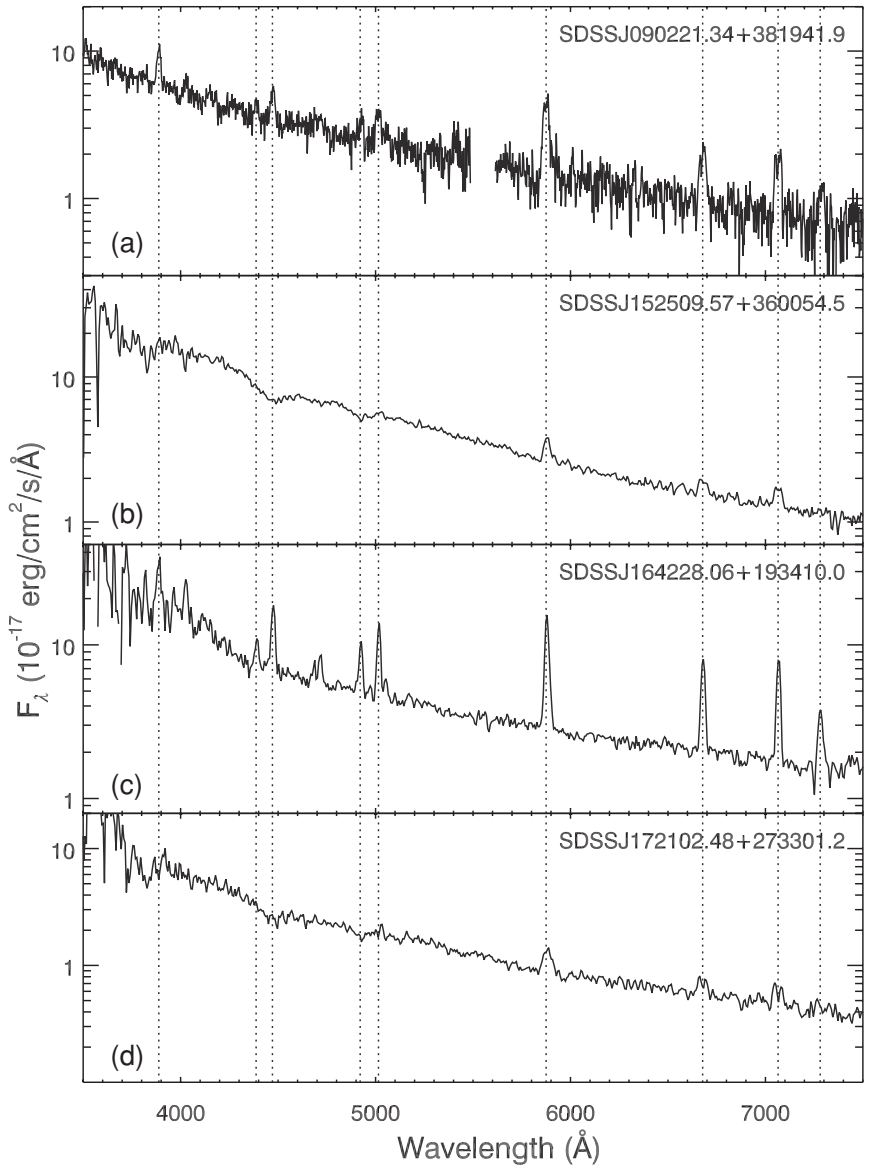

Figure 2. Dereddened low-resolution spectra of (a) SDSS J0902 (taken with P200/DBSP) (b) SDSSJ 1525, (c) SDSSJ 1642, and (d) SDSSJ 1721 (all taken with WHT/ACAM). The positions of the strongest He I lines are indicated by the dotted vertical lines.

photometry does typically not yield stable orbital period signals. In AM CVn stars, the emission lines in a spectroscopic time series are commonly observed to vary in the form of a sinusoidal "S-wave." In analogy with the bright spots seen in hydrogenrich cataclysmic variables, this S-wave is caused by the impact region where the infalling accretion stream hits the accretion disk edge, providing an emission-line source that is fixed in the binary frame. In order to search for a similar signature in our data of SDSS J0902, we combined the strongest helium lines from both nights of Keck-I/LRIS observations. We focused on the blue side of LRIS where the signal to noise is highest.

Roughly following the approach of Nather et al. (1981) we divided the combined lines into red and blue wings and calculated the ratio of the fluxes in both wings for each individual spectrum. A Lomb-Scargle periodogram of the flux ratios is shown in Figure 3. The maximum power is found at 29.8 cycles per day, corresponding to a period of 48.3 minutes. The periodogram shows the next two strongest peaks at the usual \pm 1 cycle per day aliases (see the inset of Figure 3), which are caused by the relatively short observing baseline on two consecutive nights.

Phase-folding the individual spectra of both nights on a period of 48.3 minutes produces the trailed spectrum shown in Figure 4. A clear sinusoidal S-wave with a velocity amplitude of $\approx 700 \mathrm{~km} \mathrm{~s}^{-1}$ is found. The observing baseline on the second night (see Table 1) is long enough and the $\mathrm{S}$-wave signal is strong enough that we can obtain a unique trace of the S-wave signal throughout the two-night baseline of our Keck run,

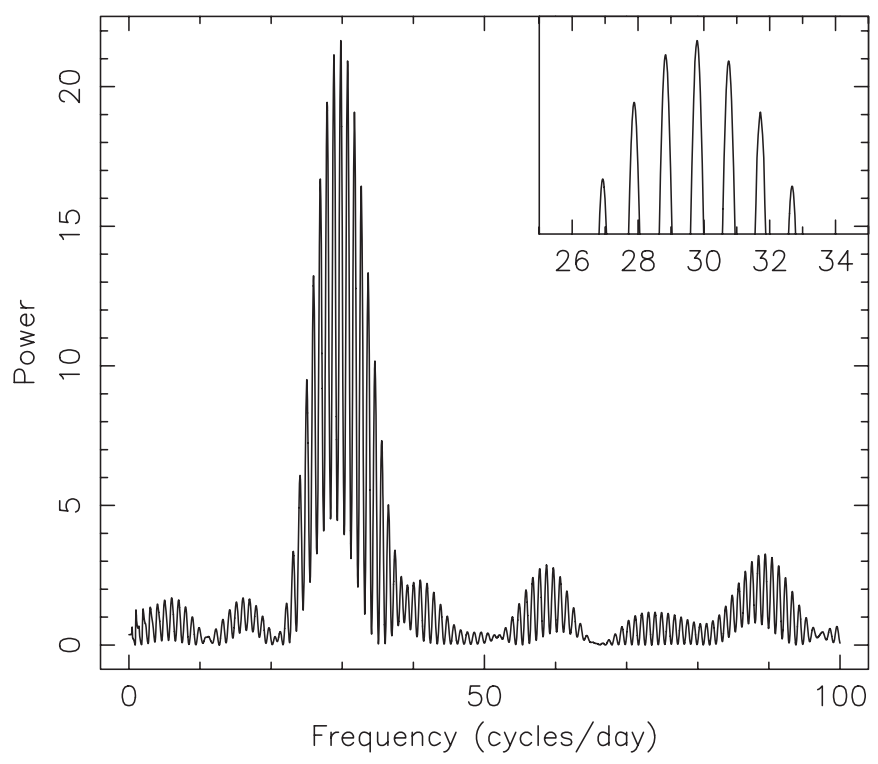

Figure 3. Lomb-Sargle periodogram of the flux ratios of the blue and red wings of the combination of the strongest He I $(\lambda \lambda 3888,4026,4471,4921,5015)$ emission lines in the blue side of the Keck-I/LRIS spectra of SDSS J0902. A zoom around the peak (inset) shows the typical aliases at \pm 1 cycle per day.

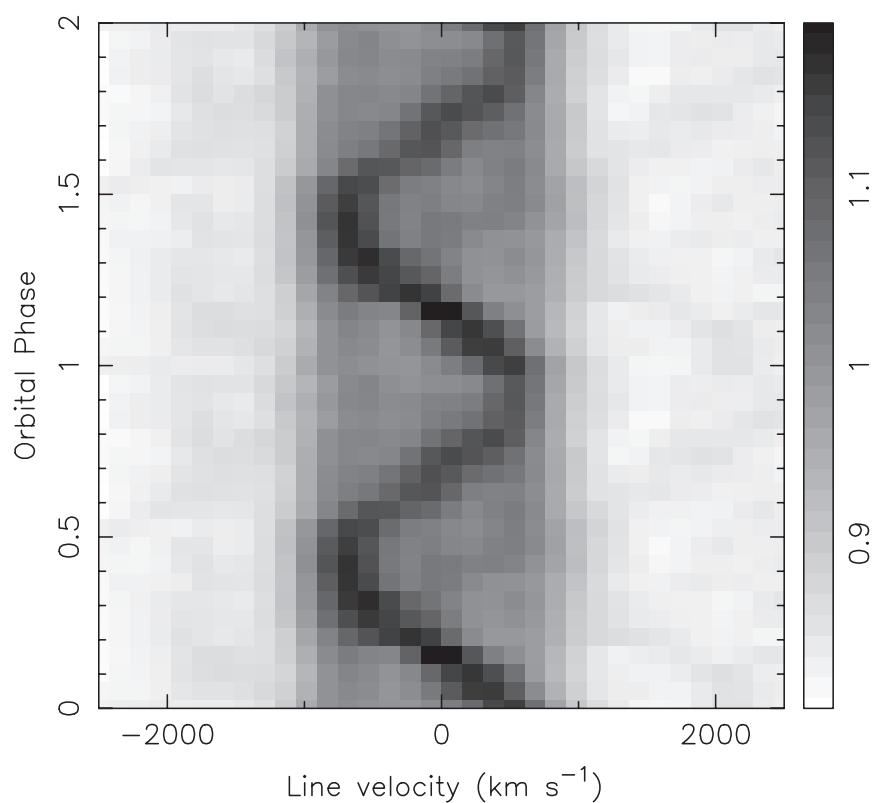

Figure 4. Trailed spectrum of the combined He emission lines in the blue arm spectra of SDSS J0902, phase-folded on the 48.31 minutes period. The zero phase is arbitrary. The gray- scale indicates the relative flux densities. An arbitrary zero phase of $\mathrm{HJD}=2454858.049150$ has been used.

without losing count of the orbital cycles. To accurately track the phase of the S-wave signal, we back-projected the spectra into a Doppler tomogram (Marsh \& Horne 1988), transforming the Swave emission into a localized emission "bright spot" (Figure 5). Fine-tuning of the phase-folding period to eliminate the phase drift of the bright spot gives an orbital period $P_{\text {orb }}=48.31 \pm$ 0.08 minutes. The error on the orbital period is obtained from an estimate of the bright-spot phasing accuracy due to statistical and systematic errors, as discussed in Roelofs et al. (2007b).

\subsection{Average Spectrum of SDSS J0902}

In order to generate an averaged, high signal-to-noise spectrum of SDSS J0902, we combined all LRIS exposures using 
Table 2

GALEX GR5 and SDSS DR7 Photometry and He I $\lambda 5876$ Equivalent Width of the New AM CVn Stars

\begin{tabular}{ccccccccccc}
\hline \hline SDSS $\ldots$ & $\mathrm{FUV}_{\mathrm{AB}}$ & $\mathrm{NUV}_{\mathrm{AB}}$ & $u$ & $g$ & $r$ & $i$ & $\mathrm{~A}_{(\mathrm{g})}$ & $-\mathrm{EW}$ \\
\hline $\mathrm{He} \mathrm{I} \lambda 5876(\AA)$
\end{tabular}

Notes. Photometry and $g$-band extinction are given in magnitudes. Numbers in parentheses indicate the errors in the corresponding number of last digits.

${ }^{a}$ Visual inspection of the GALEX All-sky Imaging Survey image AIS_85_sg82 puts doubts on the GR5 cataloged detection, as confirmed by the non-detection in a second visit (AIS_85_sg72).

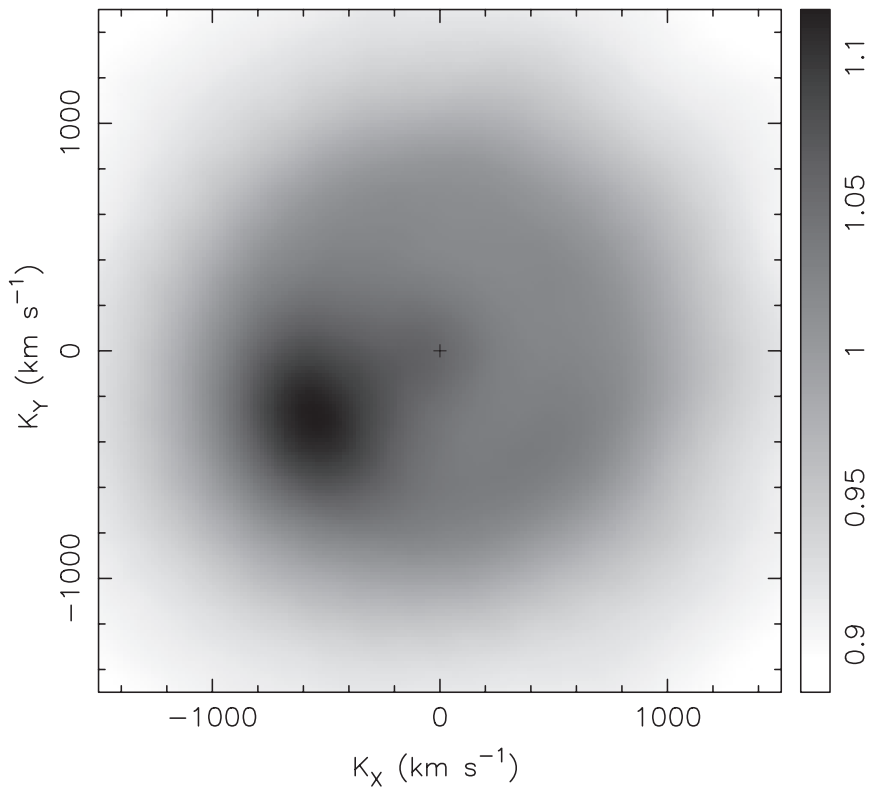

Figure 5. Linear back-projection Doppler tomogram of the combined SDSS J0902 He emission lines in the blue arm spectra taken with Keck-I/ LRIS, projected on the 48.31 minutes period. The gray- scale indicates the relative flux densities. Zero phase as in Figure 4.

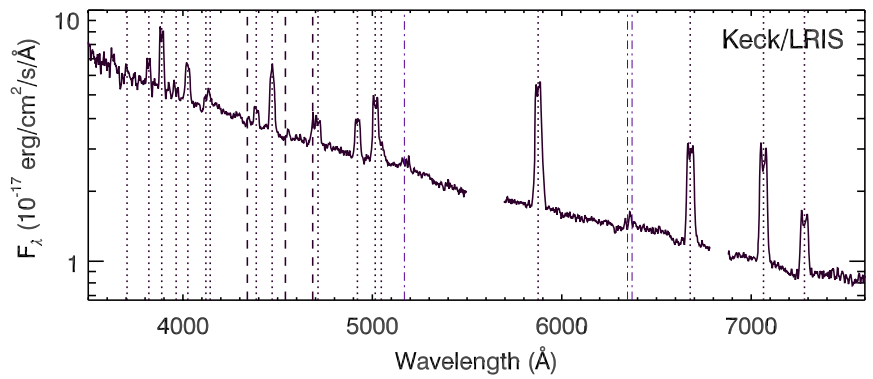

Figure 6. Average Keck-I/LRIS spectrum of SDSS J0902. Dotted and dashed lines mark the He I and He II emission features, respectively. The locations of Fe II ( $\lambda 5169)$ and Si II $(\lambda 6347,6371)$ in emission are indicated by the dash-dotted lines.

optimal weights (Figure 6). As already indicated by the DBSP spectrum (Figure 2(a)), SDSS J0902 exhibits a blue continuum dominated by strong emission of neutral helium, typical for AM $\mathrm{CVn}$ stars. In addition, the LRIS spectrum reveals singly ionized helium ( $\lambda 4686$ ) as well as weak components of Fe II $\lambda 5169$ and Si II $\lambda 6347+6371$. These features are commonly observed in AM CVn stars (e.g., Groot et al. 2001; Roelofs et al. 2006a), and are expected from a donor star with solar abundances of heavy metals (Marsh et al. 1991). We note the absence of significant DB white dwarf absorption (as seen in, e.g., SDSS J1240-0159, Roelofs et al. 2005) underlying the helium emission lines. There

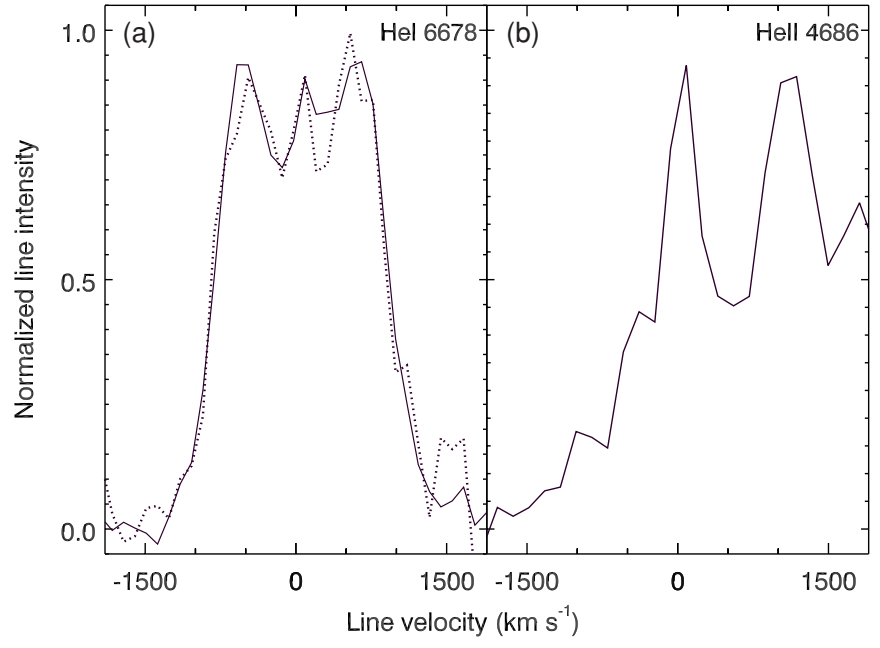

Figure 7. Emission line profiles of SDSS J0902. Left: He I 6678 shows a clear triple-peaked shape in the averaged spectrum (solid line) as well as in a single 180 s exposure (dotted line). Right: He II 4686 has a Gaussian profile. The second peak at $+1000 \mathrm{~km} \mathrm{~s}^{-1}$ corresponds to the blue wing of He I 4713 .

are two known AM CVn stars with comparable orbital period: GP Com $\left(P_{\text {orb }}=46.5\right.$ minutes; Nather et al. 1981 $)$ and SDSS $\mathrm{J} 1411+4812\left(P_{\text {orb }}=46 \pm 2\right.$ minutes; Roelofs 2007). Overall the spectrum of SDSS J0902 is particularly reminiscent of that of SDSS J1411+4812 (Anderson et al. 2005). It shows similar metal lines and He line equivalent widths (e.g., He I 5876 $\mathrm{EW}_{\mathrm{J} 0902}=79 \pm 3 \AA$ (see Table 2 ) versus $\mathrm{EW}_{\mathrm{J} 1411} \approx 65 \AA$; Anderson et al. 2005). In contrast, the high abundance of nitrogen in GP Com is not seen in SDSS J0902, which suggests that the $\mathrm{CNO}$ cycle is less prominent in the latter.

The He I emission lines, averaged as well as in single exposures, show the classical double-winged profile indicative of an accretion disk (Figure 7(a)). In addition, we detect a third emission component near zero velocity, similar to that seen in many other AM CVn stars. This emission spike is thought to originate from (or very near) the surface of the accreting white dwarf, as suggested by observed radial velocity shifts consistent with the accretor's expected orbital motion in GP Com (Marsh 1999; Morales-Rueda et al. 2003). It is interesting to note that the He II emission line at $\lambda 4686$ (blended with He I $\lambda 4713$, Figure 7(b)) has a Gaussian profile and a lower FWHM $\left(\approx 700 \mathrm{~km} \mathrm{~s}^{-1}\right)$ than the He I lines $\left(\approx 1200 \mathrm{~km} \mathrm{~s}^{-1}\right)$, suggesting that the He II line may largely consist of the central spike component.

An estimate of the continuum temperature of SDSS J0902 can be obtained by modeling the available photometric and spectroscopic data. A fit to the SDSS photometry taken in 2002 and the GALEX NUV measurement obtained in 2004 suggest a 

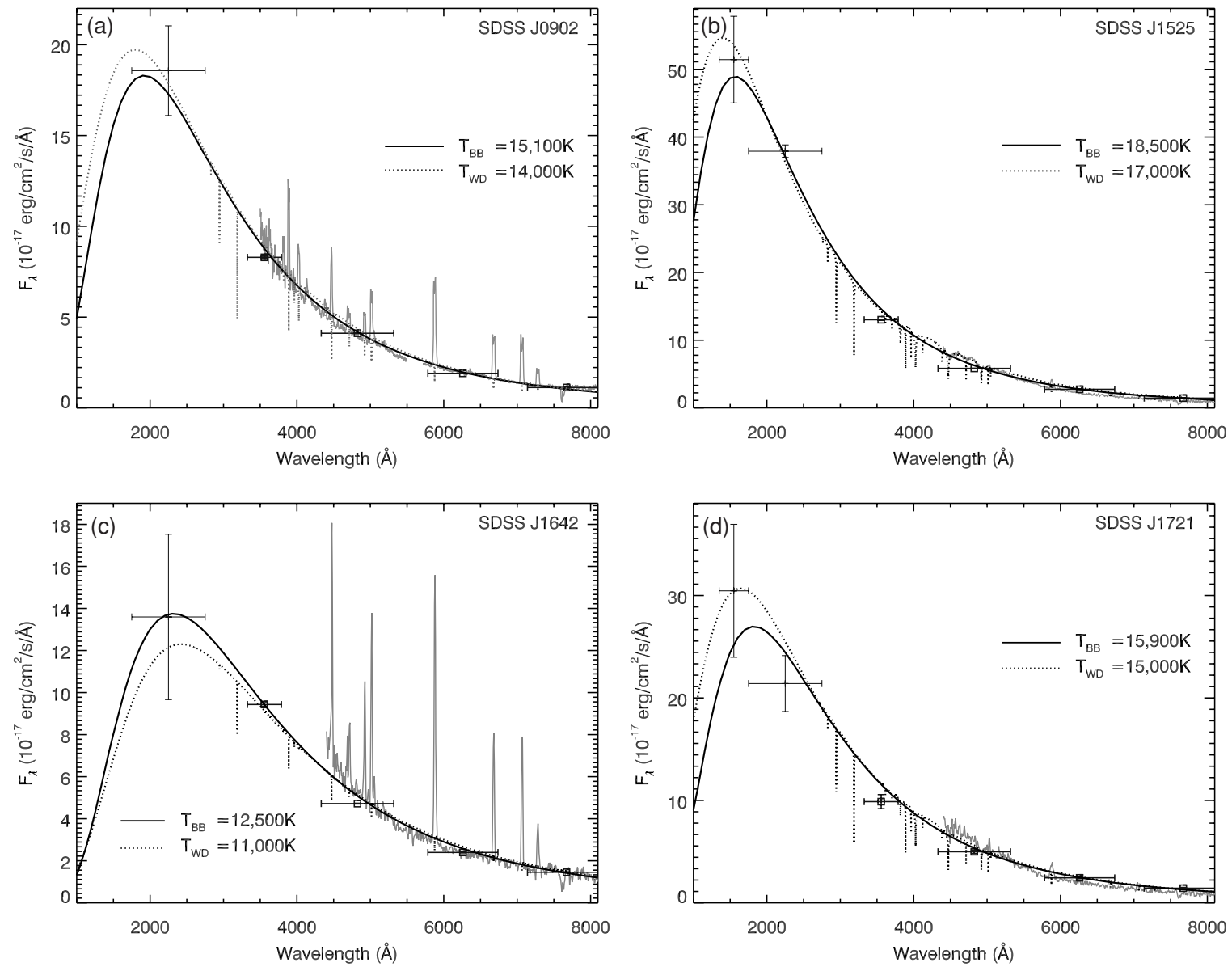

Figure 8. Flux-calibrated spectra together with photometry from GALEX GR5 (crosses) and SDSS DR7 (empty squares, see Table 2) for the four new AM CVn candidates. The photometry and spectroscopy have been corrected for Galactic foreground extinction. The solid and dotted lines indicate blackbodies and helium white dwarf atmosphere models of Koester (2008) with surface gravity $\log g=8$, respectively, fit to the available photometric data. The effective temperatures are noted in the panels. Spectroscopic observations of SDSS J0902 (a), SDSS J1525 (b), and SDSS J1721 (d) have been obtained under non-photometric conditions resulting in considerable uncertainties in the absolute flux calibration. Thus, these spectra have been scaled to the SDSS photometry for visualization purposes.

black-body (BB) with effective temperature of $T_{\text {eff }} \approx 15,100 \mathrm{~K}$ (Figure 8(a)). A similarly good match is obtained using the helium white dwarf atmosphere models of Koester (2008) with $T_{\text {eff }} \approx 14,000 \mathrm{~K}$.

At the orbital period of SDSS J0902, the accreting white dwarf is expected to contribute a large fraction $(\approx 50 \%)$ of the continuum emission, if the mass transfer rate is not significantly higher than the equilibrium rate set by gravitational-wave radiation $\left(\approx 10^{-11} M_{\odot} \mathrm{yr}^{-1}\right.$; Bildsten et al. 2006). This means that the accreting dwarf is expected to be the hottest component in the system. Interestingly, our temperature estimate is at the high end of expectations from theoretical models for the thermal evolution of the accreting white dwarf (Bildsten et al. 2006). These models predict temperatures in the range of $9000 \mathrm{~K}$ to $15,000 \mathrm{~K}$ (for primary masses from $0.65 M_{\odot}$ to $1.05 M_{\odot}$ ) at orbital periods of $\approx 48$ minutes.

Since a higher system mass leads to more accretion heating and shorter cooling time for the accretor at a given orbital period, the rather high continuum temperature in SDSS J0902 may point to a high accretor and/or donor mass. The absence of broad DB absorption lines, seen in other AM CVn stars (e.g., SDSS J1240; Roelofs et al. 2006b), suggests that the accretor cannot be much hotter than $15,000 \mathrm{~K}$, if it contributes substantially to the optical flux as expected. Assuming a 50\% contribution to the observed flux and a mass of $0.9 M_{\odot}$ (radius $0.009 R_{\odot}$ ) we derive a distance estimate of $\approx 350 \mathrm{pc}$.

\subsection{SDSS J1525, SDSS J1642, and SDSS J1721}

The two objects with the weakest emission lines, SDSS J1525 and SDSS J1721, turn over to a helium absorption-line spectrum in the blue, quite like the first Sloan-discovered AM CVn star, SDSS J1240 (Roelofs et al. 2005). BB and helium white dwarf atmosphere model fits to their SDSS and GALEX photometry (Figure 8(b) and (d)) suggest temperatures of $T_{\text {eff }}=17,000$ $18,500 \mathrm{~K}$ and $T_{\text {eff }}=15,000-15,900 \mathrm{~K}$ for SDSS J1525 and SDSS J1721, respectively. This together with the broad helium absorption lines in the spectra indicates orbital periods below $\approx 40$ minutes (Nelemans 2005). The relatively low temperature of the latter surprises given the similarly broad helium absorption lines in both systems (see Figure 2). We matched the white dwarf atmosphere models to the spectral range around the $4921 \AA$ absorption line and found a best-fit temperature of $18,000 \mathrm{~K}$ in both sources. The apparent discrepancy for SDSS J1721 suggests that another component, e.g., the accretion disk, contributes significantly to the continuum emission.

The third source, SDSS J1642, shows remarkably strong helium emission lines, comparable to CE 315 , the system with the longest orbital period $(65.1 \pm 0.7$ minutes; Ruiz et al. 2001). The absence of helium absorption and the low effective temperature of $\approx 12,000 \mathrm{~K}$ (Figure $8(\mathrm{c})$ ) suggest that SDSS $\mathrm{J} 1642$ may be of long orbital period ( $>50$ minutes) where the accretor is expected to dominate the optical flux. 
In order to approximate the distances to the three systems, we assume a more typical white dwarf mass of $0.6 M_{\odot}$ (radius $0.0123 R_{\odot}$ ) and a $50 \%$ flux contribution by the accretor. Using the temperatures estimated above, we find distances of $\approx 450 \mathrm{pc}$ (SDSS J1525, SDSS J1721) and $\approx 350$ pc (SDSS J1642).

\section{CONCLUSIONS}

We have presented four proposed new AM CVn stars and time-resolved spectroscopy confirming beyond doubt the ultra-compact binary nature of one of them, SDSS J090221.35+381941.9. This increases the total count of new systems to five, after completing the spectroscopic follow-up of approximate half of the 1500 candidates in our sample. Based on the present sample completeness (Figure 1), we expect close to eight more AM CVn stars among the remaining candidates. This already indicates a lower space density than predicted earlier (Roelofs et al. 2007a), but a detailed population study will be presented in a separate paper after the follow-up program has been completed.

SDSS J0902 is the second confirmed AM CVn star found in our ongoing spectroscopic survey of color-selected candidates from the SDSS, after SDSS J0804 (Roelofs et al. 2009), and has an orbital period of $P_{\text {orb }}=48.31 \pm 0.08$ minutes. With an apparent temperature of $T_{\text {eff }} \approx 14,000 \mathrm{~K}$, its optical/UV spectrum is at the high end of expectations from theoretical cooling models, assuming gravitational-wave-driven evolution. This is becoming a common observational feature and may suggest large component masses in many AM CVns, or angular momentum losses additional to gravitational radiation.

At $g=20.23$ mag $_{\mathrm{AB}}$, SDSS J0902 is near the faint magnitude limit of our sample. Our successful follow-up shows that phaseresolved spectroscopy of faint systems can be obtained at 8-10 m class telescopes within a modest amount of time, thanks to the strong S-wave signals typical of the emission-line AM CVn stars. Similar observations of the other new candidates are encouraged to confirm their ultra-compact binary nature and build up the orbital period distribution, which holds valuable information about the Galactic population as a whole and the physics governing its evolution (e.g., Deloye et al. 2007).

A.R. acknowledges support through NASA grant NNX08AK66G. G.H.A.R. is supported by NWO Rubicon grant 680.50.0610. D.S. acknowledges a STFC Advanced Fellowship. This paper is based on data obtained at the W. M. Keck Observatory, which is operated as a scientific partnership among the California Institute of Technology, the University of California, and the National Aeronautics and Space Administration (NASA). The Observatory was made possible by the generous financial support of the W. M. Keck Foundation. We also acknowledge use of the Palomar Hale-5 m telescope operated by the California Institute of Technology and Palomar Observatory; the Nordic Optical Telescope and the William Herschel Telescope, La Palma; and the GALEX public archive. We are grateful to D. Koester for kindly making available his white dwarf atmosphere models.

\section{REFERENCES}

Anderson, S. F., et al. 2005, AJ, 130, 2230

Bildsten, L., Shen, K. J., Weinberg, N. N., \& Nelemans, G. 2007, ApJ, 662, L95

Bildsten, L., Townsley, D. M., Deloye, C. J., \& Nelemans, G. 2006, ApJ, 640, 466

Cropper, M., Harrop-Allin, M. K., Mason, K. O., Mittaz, J. P. D., Potter, S. B., \& Ramsay, G. 1998, MNRAS, 293, L57

Deloye, C. J., Bildsten, L., \& Nelemans, G. 2005, ApJ, 624, 934

Deloye, C. J., Taam, R. E., Winisdoerffer, C., \& Chabrier, G. 2007, MNRAS, 381,525

Groot, P. J., Nelemans, G., Steeghs, D., \& Marsh, T. R. 2001, ApJ, 558, L123

Israel, G. L., et al. 2002, A\&A, 386, L13

Koester, D. 2008, in Mem. Soc. Astron. Ital., Lectures at the School of Astrophysics F. Lucchin, Tarquinia (arXiv:astro-ph/0812.0482)

Marsh, T. R. 1999, MNRAS, 304, 443

Marsh, T. R., \& Horne, K. 1988, MNRAS, 235, 269

Marsh, T. R., Horne, K., \& Rosen, S. 1991, ApJ, 366, 535

Morales-Rueda, L., Marsh, T. R., Steeghs, D., Unda-Sanzana, E., Wood, J. H., \& North, R. C. 2003, A\&A, 405, 249

Nather, R. E., Robinson, E. L., \& Stover, R. J. 1981, ApJ, 244, 269

Nelemans, G. 2005, in ASP Conf. Ser. 330, The Astrophysics of Cataclysmic Variables and Related Objects, ed. J.-M. Hameury \& J.-P. Lasota (San Francisco, CA: ASP), 27

Nelemans, G., Portegies Zwart, S. F., Verbunt, F., \& Yungelson, L. R. 2001, A\&A, 368, 939

Nelemans, G., Yungelson, L. R., \& Portegies Zwart, S. F. 2004, MNRAS, 349, 181

Oke, J. B. 1990, AJ, 99, 1621

Oke, J. B., \& Gunn, J. E. 1982, PASP, 94, 586

Oke, J. B., et al. 1995, PASP, 107, 375

Podsiadlowski, P., Han, Z., \& Rappaport, S. 2003, MNRAS, 340, 1214

Roelofs, G. H. A. 2007, PhD thesis, Radboud Univ. Nijmegen, The Netherlands

Roelofs, G. H. A., Groot, P. J., Benedict, G. F., McArthur, B. E., Steeghs, D., Morales-Rueda, L., Marsh, T. R., \& Nelemans, G. 2007a, ApJ, 666, 1174

Roelofs, G. H. A., Groot, P. J., Marsh, T. R., Steeghs, D., Barros, S. C. C., \& Nelemans, G. 2005, MNRAS, 361, 487

Roelofs, G. H. A., Groot, P. J., Marsh, T. R., Steeghs, D., \& Nelemans, G. 2006a, MNRAS, 365, 1109

Roelofs, G. H. A., Groot, P. J., Nelemans, G., Marsh, T. R., \& Steeghs, D. 2006b, MNRAS, 371, 1231

Roelofs, G. H. A., Groot, P. J., Nelemans, G., Marsh, T. R., \& Steeghs, D. 2007b, MNRAS, 379, 176

Roelofs, G. H. A., Nelemans, G., \& Groot, P. J. 2007d, MNRAS, 382, 685

Roelofs, G. H. A., et al. 2009, MNRAS, 394, 367

Ruiz, M. T., Rojo, P. M., Garay, G., \& Maza, J. 2001, ApJ, 552, 679

Schlegel, D. J., Finkbeiner, D. P., \& Davis, M. 1998, ApJ, 500, 525

Stroeer, A., Vecchio, A., \& Nelemans, G. 2005, ApJ, 633, L33

York, D. G., et al. 2000, AJ, 120, 1579 